\title{
Compression and Visualization of the Operational Parameters Archive
}

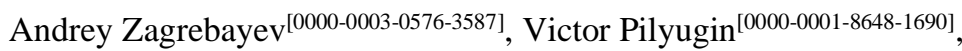 \\ Stanislav Ten ${ }^{[0000-0001-9279-1732]}$ \\ National Research Nuclear University MEPhI, Kashirskoe hwy 31, 115409, Moscow, Russia \\ \{amzagrebayev, vvpilyugin\}@mephi.ru \\ tenstanislav@email.com
}

\begin{abstract}
This article describes the mathematical apparatus for express analysis of the archive of operational parameters. The developed algorithms based on the methods of reducing the dimension of the space of variables and the "Chernoff Faces" method allow visualizing the dynamics of changes in generalized limiting parameters, as well as visually determining the approximation or intersection of permitted values by parameters, which in turn can provide scientific and practical use in improving quality of operational personnel work and analysis of situations requiring additional attention and more detailed analysis.
\end{abstract}

Keywords: Operational Parameters Archive, Scientific Visualization, Data Analysis.

\section{Introduction}

The safe operation of powerful nuclear power reactors is ensured by the availability of information and computer systems that make it possible to measure, calculate and control the most important parameters of a nuclear power unit [1-4].

At power units with RBMK reactors such a system is called "SKALA", which allows controlling about 10,000 parameters in almost real time mode. This information is partially visualized on a unit control board and enables operators to efficiently and safely manage a nuclear power unit.

Moreover, in addition to the functions of directly providing information for the operational management of the technological process, the information and computer complex archives the current measured and calculated parameters. This enables a posteriori analysis of the quality of process control in order to obtain new scientific and practical results.

List as an example some of the tasks that require knowledge and analysis of the history of the power unit's behavior:

Copyright $(C 2020$ for this paper by its authors. Use permitted under Creative Commons License Attribution 4.0 International (CC BY 4.0). 
2 A. Zagrebayev et al.

- Diagnosis of emergency situations at power unit, evaluation of operational staff work quality;

- evaluation of efficiency of held events for algorithm and control systems improvement;

- creation of real data information base for development of mathematical software of a training apparatus;

- creation of adapting mathematical models of processes, ongoing in nuclear power installation;

- creation of self-organizing programs of "operator advisor" type, using work experience of operational staff in regular and emergency situations (solving problems of artificial intelligence);

- identification of reasons of separate items of reactor core and main technological equipment break down (solving problems of predictive analytics).

\section{Problem definition}

Depending on the problem, requirements for pattern, type, amount of stored information and also for the level of its specification may significantly differ. For example, to solve the problem of assessing the quality of work of operational staff, it is necessary to store information on the type and number of monitored parameters, the values of which are beyond the limits established by the regulation, the number and type of operator's effects on the control object (movement of control and protection systems (for CPS), coolant flow rate adjustment, etc.), the degree of spatial stability of the threedimensional energy release field, etc. While solving the problem of identification of break down reasons, for example, of fuel elements and a channel, information for the period from several days to several years may be needed. In this case a behavior backstory of such parameters as each channel capacity, its power generation, coolant flow rate through the channel, fuel elements' burst can detection system data and integrity control of technological channels [1], linear load on a fuel element, stock before the heat transfer crisis, number of transpositions of fuel cartridges and etc. are of interest.

However, despite the fact that the above problems are actually of different types, power unit operational parameters archive should enable each problem to be solved and moreover, should be an information database for solving newly arising problems. At the current level of development of computer systems at nuclear power plants, it is possible to organize the storage of all experimental and calculated information with high detail in time over a long period of operation, however, this raises problems with express analysis of a large amount of data. The way out of this situation can be the use of the method of scientific visualization, when the initial analyzed data is assigned to one or another form of its graphic interpretation, which can subsequently be analyzed visually, and the results of the analysis of this graphic interpretation are then interpreted in relation to the original data.

A modern storage facility for parameters of a nuclear power unit with an RBMK reactor and a special created module for visualizing archive data in a user-friendly form 
for viewing the archive database from remote workstations are described in [5]. This module also serves for easy export of data for subsequent analysis and calculations.

The module interface is presented in Fig. 1. In the main window of the program the current state of the database is analyzed and a list of available "time slices" is generated. The header of the main window displays information about the time of the last update is presented. On the toolbar there is a button to update the list of available "time slices" manually. In the center of the program window the cartogram of the parameter selected from the list is displayed. Periodically (by default every 30 seconds) the program automatically updates the list of available "time slices".

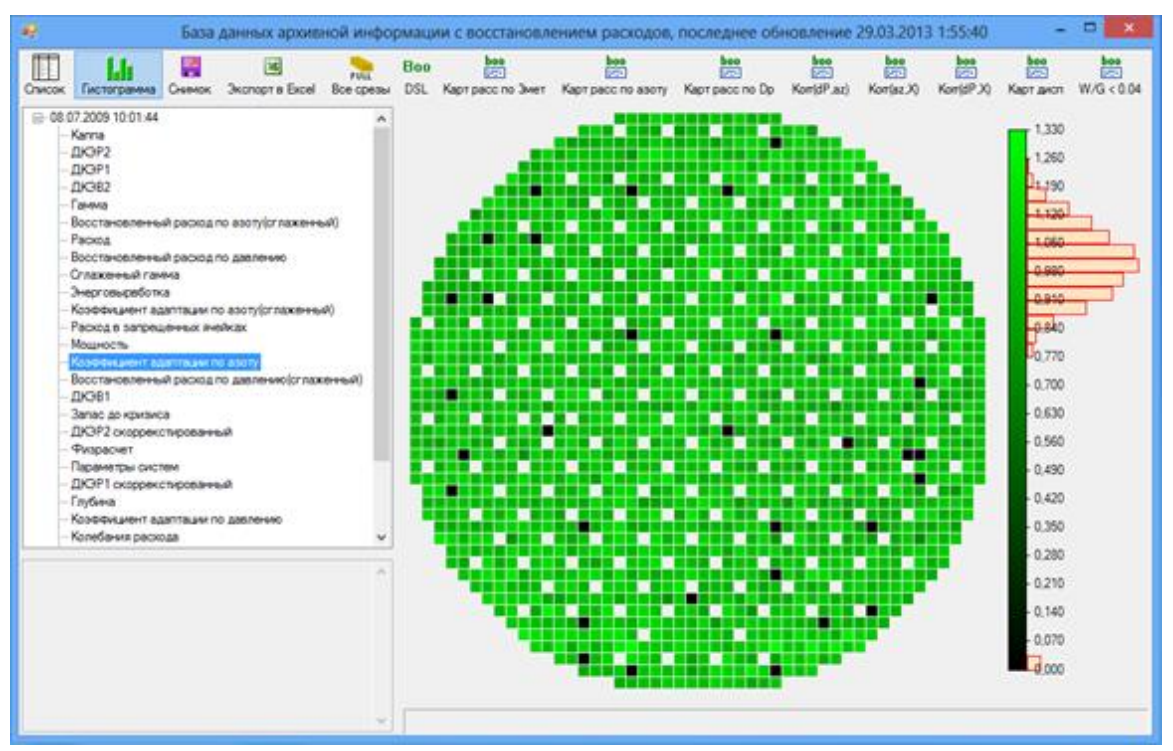

Fig. 1. The window of the visualization module after selecting the required information

From the "SKALA-MICRO" system such parameters as core fueling, the coolant flow rate in each channel, information about the position of control rods, the readings of the neutron flux sensors and the estimated parameters reactor power, energy-producing of fuel assembly and reactivity margin are extracted.

Despite the advantages of the visualization module above, that are the presentation of information in the form of cartograms and graphs of detailed behavior of local parameters, there are some problems from the express analysis point of view of the parameters' temporal behavior. It would be desirable to visualize the generalized parameters of a nuclear power unit containing the most important information about the technological process in the integral.

This opportunity is fundamentally possible, since there really are single objects, for example, neutron fields, graphite masonry temperatures, etc., although they are measured by sensors discretely located in space. This is on the one hand. On the other hand, the archive of operational parameters contains parameters correlated with each other, since different control systems provide dissimilar information, but about the same object. 


\section{A. Zagrebayev et al.}

Thus, there is, firstly, a need to compress information and obtain a number of parameters, the physical meaning of which simplifies the visual analysis of the process history, and secondly, the development (or application) of modern methods of visualization of multidimensional objects and processes.

This work illustrates the proposed approach with specific examples.

\section{$3 \quad$ Visualization of multidimensional data}

It is proposed to use the least squares method [6] and the method of selecting significant ordinates [7] as the algorithms for compressing information on the neutron field. The first one is used to compress intra-reactor information in space, and the second one is to compress single and integral parameters in time.

Let us briefly consider the technique of compressing information in space. Suppose that the field of some operational parameter of the reactor core is described by a spatiotemporal function $\Phi(\vec{r}, t)$. Approximate the readings of discretely located sensors based on the least squares method.

Represent the function $\Phi(\vec{r}, t)$ in the form (1), and seek a solution based on the minimum condition for functional (2).

$$
\begin{gathered}
\Phi(\vec{r}, t)=\sum_{i=1}^{m} B_{i}(t) \varphi_{i}(\vec{r}) ; \\
F=\sum_{i=1}^{m}\left(\Phi\left(\vec{r}_{i}, t\right)-C_{i}\right)^{2} \rightarrow \min ,
\end{gathered}
$$

here $C_{i}$ is the value of the operational parameter at the points where the sensors are installed; $\vec{r}_{i}$ - coordinates of the reactor core points where the sensors are installed; $\varphi_{i}(\vec{r}), i=1, \ldots, m$ - a number of linearly independent functions that are selected in advance; $B_{i}(t)$ - time coefficients to be found.

With this formulation of the problem, the solution is obtained in the form

$$
\vec{B}=\left(S^{T} S\right)^{-1} S^{T} \vec{C}, \vec{B}=M \vec{C}, M=\left(S^{T} S\right)^{-1} S^{T},
$$

where $\vec{C}=\left(\begin{array}{c}C_{1} \\ C_{2} \\ \ldots \\ C_{N}\end{array}\right)$ is a parameter measurement vector;

$\vec{B}=\left(\begin{array}{c}B_{1} \\ B_{2} \\ \ldots \\ B_{m}\end{array}\right)$ is a vector of unknown coefficients; 
$S=\left(\begin{array}{cccc}\varphi_{1}\left(\vec{r}_{1}\right) & \varphi_{2}\left(\vec{r}_{1}\right) & \ldots & \varphi_{m}\left(\vec{r}_{1}\right) \\ \varphi_{1}\left(\vec{r}_{2}\right) & \varphi_{2}\left(\vec{r}_{2}\right) & \ldots & \varphi_{m}\left(\vec{r}_{2}\right) \\ \ldots & \ldots & \ldots & \ldots \\ \varphi_{1}\left(\vec{r}_{N}\right) & \varphi_{2}\left(\vec{r}_{N}\right) & \ldots & \varphi_{m}\left(\vec{r}_{N}\right)\end{array}\right)$ is a matrix of values of decomposition functions at measurement points.

Thus, instead of the initial $N$ values of the measurement vector $\vec{C}, \mathrm{~m}$ values of the $B_{i}$ coefficients are obtained. The number of $B_{i}$ coefficients is 1-2 orders of magnitude smaller than the number $N$, and satisfactory accuracy is achieved.

The choice of decomposition functions during data compression by the least squares method may be arbitrary in the general case, but in order to achieve maximum approximation accuracy, it is desirable to choose them most closely describing the behavior of the reactor parameters. On the one hand, it is known [1] that such functions are eigenfunctions for solving equations describing the dynamics of the parameters under consideration. On the other hand, finding the exact solution that describes the behavior of the parameters in the reactor is a difficult mathematical task, since a nuclear power reactor is a complex object with distributed parameters.

In [8] there is the following method of experimental determination of the best set of test functions, based on the transition from the initial expansion of the parameters in some functions that approximately describe their behavior to the canonical expansion obtained from the original.

It is necessary to find such decomposition functions $\left\{\varphi_{i}^{\prime}(\vec{r})\right\}$ in order to present the function $\Phi(\vec{r}, t)$ in the form:

$$
\Phi(\vec{r}, t)=\sum_{i=1}^{M} V_{i}(t) \varphi_{i}^{\prime}(\vec{r}),
$$

in this case $V_{i}$ are not correlated with each other, in contrast to $B_{i}$ in formula (1).

$M\left[V_{i} V_{j}\right]=0$ with $i \neq j ; M\left[V_{i} V_{j}\right]=D\left[V_{j}\right]=D_{j}$ with $i=j$, and also $M\left[V_{i}\right]=0$.

The new set of functions is calculated using the following formulas:

$$
\begin{aligned}
& \left\{\begin{array}{l}
\widetilde{\Phi}_{i}(\vec{r})=\varphi_{i}(\vec{r})+\sum_{s=i+1}^{N} a_{s i} \varphi_{s}(\vec{r}), i=1, \ldots, N-1 ; \\
\widetilde{\Phi}_{N}(\vec{r})=\varphi_{N}(\vec{r})
\end{array}\right. \\
& \left\{\begin{array}{l}
a_{i 1}=\frac{K_{i 1}}{K_{11}} \\
a_{i p}=\frac{1}{D_{p}}\left[K_{i p}-\sum_{\lambda=1}^{p-1} a_{i \lambda} a_{p \lambda} D_{\lambda}\right] \\
i=\overline{2, N} \\
p=\overline{2, i-1}
\end{array}\right.
\end{aligned}
$$

where $D_{p}=K_{p p}-\sum_{\lambda=1}^{p-1}\left(a_{p \lambda}\right)^{2} D_{\lambda}$.

$K_{i j}$ values are correlation moments: $K_{i j}=M\left[\left(B_{i}-m_{i}\right)\left(B_{j}-m_{j}\right)\right]$.

Here $m_{j}=\frac{\sum_{i=1}^{N} B_{j}^{(i)}}{N}, N$ is the sample size.

The desired function $\Phi(\vec{r}, t)$ is expressed as following:

$$
\Phi(\vec{r}, t)=\sum_{i=1}^{M} m_{i} \varphi_{i}(\vec{r})+\sum_{i=1}^{M} V_{i} \widetilde{\Phi}_{i}(\vec{r}) .
$$




\section{A. Zagrebayev et al.}

To find the coefficients, it is necessary to apply the least squares method described above.

Due to the fact that the canonical decomposition obtained by the described method allows the best description of the macro-field of reactor parameters, it is advisable to recalculate the functions $\Phi(\vec{r}, t)$ in relation to the dynamic archive in order to achieve the most accurate decomposition, since in this case fewer decomposition functions will be required to fulfil the conditions for the accuracy of decomposition.

This approach to visualizing the archive of operational parameters is illustrated by the following examples.

\subsection{Motion path in principal components}

Compression of several correlated limiting parameters of the fuel channel by the method of principal components and further dynamic visualization of the state in threedimensional space.

The limiting parameters that ensure the normal operation of the fuel assembly are:

- Power of the fuel assembly;

- coolant flow rate;

- power safety factor;

- safety factor for linear load;

- fuel temperature;

- temperature of the shell of fuel elements, etc.

Exceeding the settings of at least one of the parameters leads to an unplanned decrease in emergency power. In this work, as a result of compression, three integral parameters that characterize the state of the fuel assembly are left. This state in dynamics is displayed as the movement in time of a point in three-dimensional space limited by tolerances (see Fig. 2).
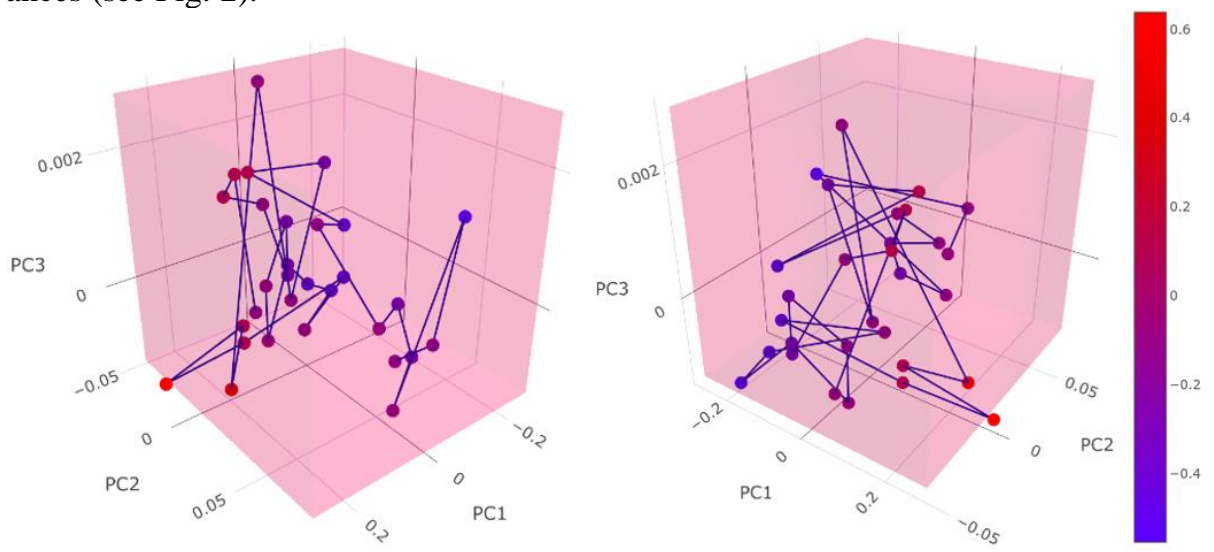
Fig. 2. Visualization of the motion path in the principal components (different angles)

\subsection{Adaptation of the "Chernoff Faces" method to the archive}

A convenient approach to visualizing the behavior of parameters is "Chernoff Faces", invented by Herman Chernoff in 1973, display multivariate data in the shape of a human face. The individual parts, such as eyes, ears, mouth and nose represent values of the variables by their shape, size, placement and orientation [9]. The idea behind using faces is that humans easily recognize faces and notice small changes without difficulty. Typically, "Chernoff Faces" are used when it is necessary to group (cluster) objects according to several criteria, or when it is necessary to analyze presumably complex relationships between variables.

Applied to the express analysis of the operational parameters archive, the Chernoff method significantly expands the range of visualized states of the reactor and the power unit as a whole.

As an example, let's use this approach to display power dynamics in local regions of the core. Local regions in this case are understood as quadrants of the reactor core.

Let $W_{(x, y)}^{t}$ be the power of the fuel assembly having coordinates on the core scheme at time $t$ (Fig. 3).

Further, the cartogram of the reactor is divided into 4 parts (quadrants), as shown in Fig. 3. Each quadrant corresponds to a set of coordinates of the channels located in this quadrant:

$$
\begin{gathered}
Q_{i}=\left\{\left(x_{1}, y_{1}\right),\left(x_{1}, y_{2}\right), \ldots,\left(x_{2}, y_{1}\right),\left(x_{2}, y_{2}\right), \ldots,\left(x_{p}, y_{q}\right), \ldots\right\}, \\
i=1, \ldots, 4-\text { quadrant number }
\end{gathered}
$$

The average power value for each quadrant is taken for each time moment $t$.

$$
\bar{W}_{i}^{t}=\sum_{\left(x_{p}, y_{q}\right) \in Q_{i}} \frac{W_{\left(x_{p}, y_{q}\right)}^{t}}{n},
$$

$n$ - number of channels in quadrant, $i=1, \ldots, 4$ - number of quadrant.

As a result, for each moment of time, each quadrant corresponds to a parameter which is the average value of power $\bar{W}_{i}^{t}$.

Then, the normalization procedure is applied for each quadrant according to the formula for the entire time slice (given time slice):

$$
X_{\text {norm }}=\frac{X-X_{\min }}{X_{\max }-X_{\min }}
$$

$X$ is the initial value is the value at the time $t, X_{\min }$ is the minimum value for the entire time slice, $X_{\max }$ is the maximum value for the entire time slice.

The normalized value is denoted by $\widehat{W}_{i}^{t}$.

At the mapping stage, 4 face characteristics were selected for visualization:

- $P_{1}$ for the tilt of the eyebrows;

- $P_{2}$ for the eye width; 
8 A. Zagrebayev et al.

- $P_{3}$ for the length of the nose;

$P_{4}$ for the bend of a smile.

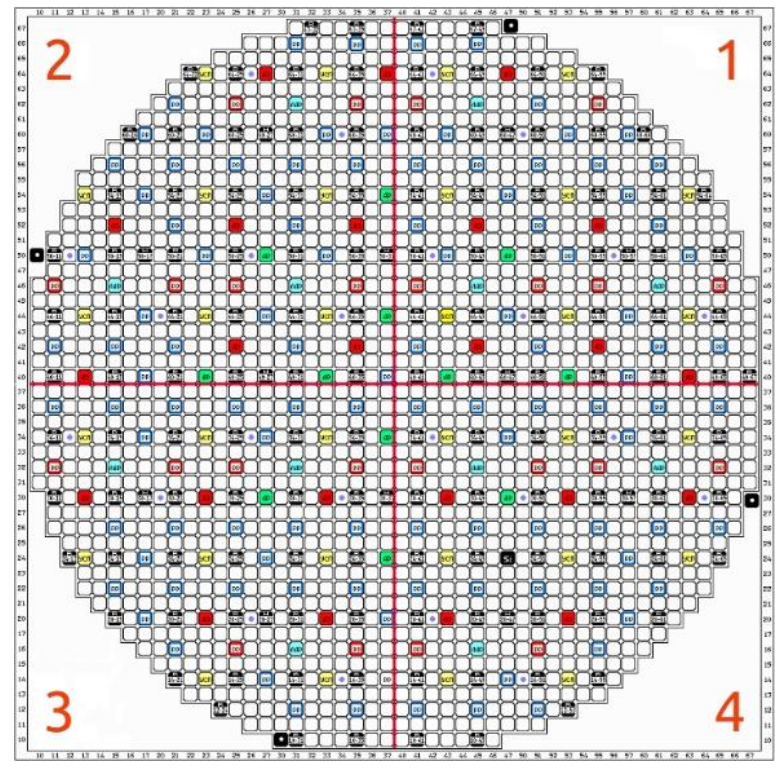

Fig. 3. Scheme of reactor core divided into quadrants

Each of the characteristics $P_{i}$ lies in the range $[0,1]$. The "average face" is displayed separately, it has the average values of the parameters for the entire time slice (the specified time slice) displayed. Fig. 4 shows an example of such a face which characteristics of 0.5 , i.e.

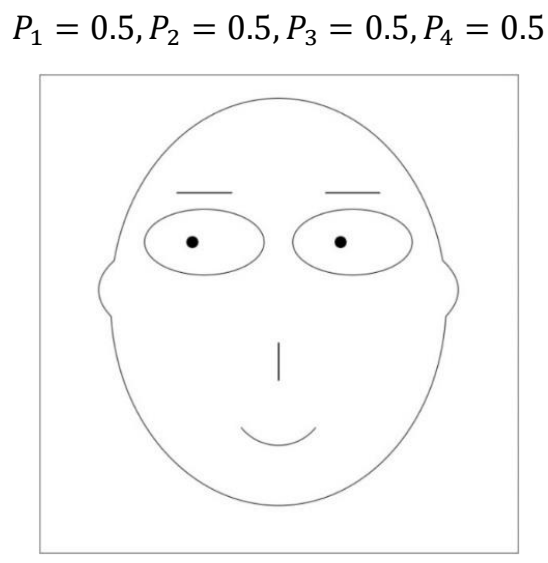

Fig. 4. "Average face" 
Further, each characteristic is associated with a normalized quadrant average power value:

$$
P_{i}=\widehat{W}_{i}^{t}
$$

and for each point in time $t$, a corresponding face is displayed.

The final result of visualization for power using the "Chernoff Faces" method is observed in Fig. 5. A similar procedure was carried out for the coolant flow rate (Fig. $6)$.

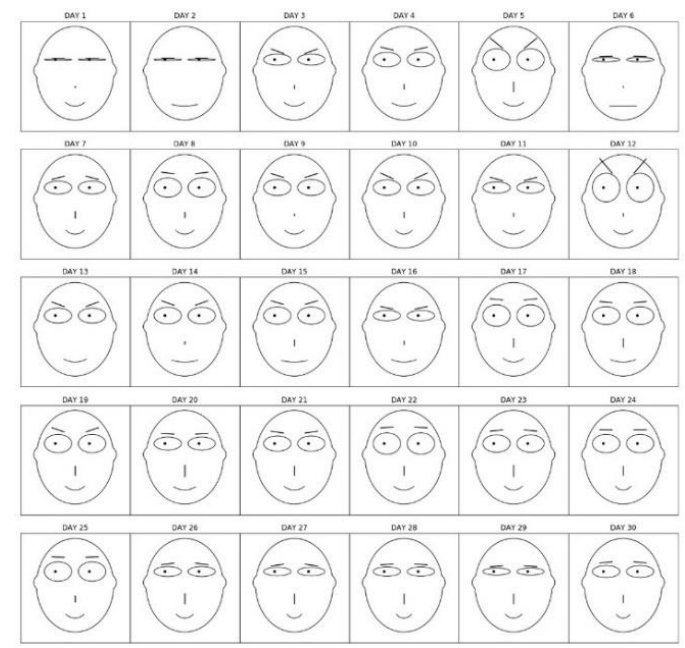

Fig. 5. Visualization results (Power)

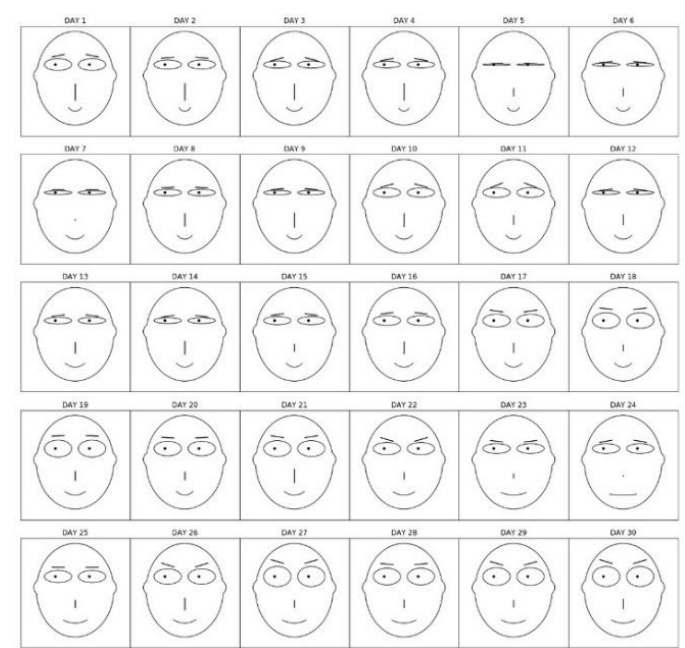

Fig. 6. Visualization results (Flow rate). 
10 A. Zagrebayev et al.

Analyzing the obtained results, it is possible to draw conclusions when some of the parameters deviate strongly from their average values, when maximums or minimums are reached, or implicit relationships between the parameters (when visualizing more parameters) can be found. The result obtained at this stage is intermediate and is used as a demonstration of possible options for visualizing the archive. Such an approach, while using a larger number of parameters, would visually highlight clusters of similar facial expressions or find hidden dependencies of the parameters among themselves.

\subsection{Temporal Networks}

Temporal Networks is another popular method for visualizing structured, dynamically changing data over time.

A temporal network, also known as a time-varying network, is a network which links are active only at certain points in time. Each link carries information on when it is active, along with other possible characteristics such as weight. Time-varying networks are of particular relevance to spreading processes, like the spread of information and disease, since each link is a contact opportunity and the time ordering of contacts is included [10].

At the moment, work is underway in the field of studying the visualization methods acceptable for use with respect to the existing archive, including the Temporal Networks method that can be used to visualize the interaction of parameters in time and can help understand, predict and optimize the behavior of system. Representation of data in the form of graphs of vertices connected by edges could reveal new non-trivial patterns of changes in parameters, possible correlations, and also could be used for clustering. At the same time, it becomes possible to assess how local patterns interact and produce global behavior, which is one of the main tasks of archive analysis.

Nowadays, Temporal Networks is actively used in the scientific community, while there is already corresponding software for working with dynamically changing data, as well as many articles on the application of this method to various data.

\section{Conclusion}

Currently, on the basis of the proposed methods, a computer software package that provides flexible customization of the desired visualization has been developed. The program module includes components for export and data processing, two-dimensional and three-dimensional visualization with the specified settings. The software is implemented using modern effective data analysis tools and provides convenient user interaction functionality.

The proposed software can be used both by the operational staff of the NPP as an auxiliary one in order to increase the efficiency of monitoring the operation of the power unit, and also in order to analyze the existing archive database.

It is planned to continue work on improving the quality and stability of the developed software package, as well as expanding the functionality and adding new features. 


\section{References}

1. Dollezhal' N.A., Emel'yanov I.Ya. Kanal'nyi yadernyi ehnergeticheskii reaktor. [Channel Nuclear Power Reactor] Atomizdat Publ., 1980.

2. M.A. Abramov, V.I. Avdeev, E.O. Adamov et al.Edited by Yu.M. Cherkashova. Kanal'nyi yadernyi ehnergeticheskii reaktor RBMK.[Channel Nuclear Power Reactor RBMK] GUP NIKIEhT Publ., 2006. 632 p.

3. Ovchinnikov F.Ya., Golubev L.I., Dobrynin V.D., Klochkov V.I., Semenov V.V., Tsybenko V.M. Ehkspluatatsionnye rezhimy vodo-vodyanykh ehnergeticheskikh yadernykh reaktorov. [Operational modes of water-cooled nuclear power reactors] Atomizdat Publ., 1975.

4. Nuclear Power Engineering. Problems. Solution / Edited by M.N. Strikhanov. - Part 1. - M.: Social Forecasts and Marketing Center, 2011. - 424 p.

5. A.M. Zagrebayev, R.N. Ramazanov, Nuclear Reactor RBMK Archive Data Visualization. Scientific Visualization, 2015, Q.2, V.7, Num. 2, pp. 1-11

6. Yurova L.N., Naumov V.I., Savander V.I., Zagrebayev A.M. Kompaktnoe predstavlenie vynutrireaktornoi informatsii o potoke neitronov / Fizika yadernykh reaktorov. [Compact representation of in-reactor neutron flux information. / Physics of nuclear reactors.] Atomizdat Publ., 1975, n.4, pp.19-23

7. Alimov A.L., Shchadilov A.E. Optimal'noe adaptivnoe szhatie tsifrovykh soobshchenii po algoritmu kusochno-lineinoi approksimatsii. [Optimal adaptive compression of digital messages using piecewise linear approximation algorithm] Avtometriya Publ., 1983, №3, pp.1418.

8. A.M. Zagrebayev, V.A. Nasonova, N.V. Ovsyannikova. Matematicheskoe modelirovanie yadernogo reaktora pri sluchainykh vozmushcheniyakh tekhnologicheskikh parametrov. [Mathematical modeling of a nuclear reactor with random perturbations of technological parameters] NRNU MEPhI Publ., 2009. - 400 p.

9. Wikipedia contributors. Chernoff face. Wikipedia, The Free Encyclopedia. Retrieved May 5, 2020. Available at:

https://en.wikipedia.org/w/index.php?title=Chernoff_face\&oldid=958878449

10. Wikipedia contributors. Temporal network. Wikipedia, The Free Encyclopedia. Retrieved August 11, 2020. Available at:

https://en.wikipedia.org/w/index.php?title=Temporal_network\&oldid=960041057 